\title{
A New Synthetic Route for Natural Products with the Pyranobenzophenone Moiety: Clusiacitran A, Vismiaphenone B, Isovismiaphenone B, and Myrtiaphenone B
}

\author{
Yong Rok Lee, Jung Hee Kim, Chul Soon Yong, Jong Sub Im, ${ }^{\dagger}$ and Won Seok Lyoo \\ School of Chemical Engineering and Technolog, Yeungham Lniversity, Gyeongsan 71 2-749, Korea. E-mail: wrlee anu ac.kr \\ College of Pharmacy, Yetngnam Lniversitw, Gveongsan 712-749, Korea \\ ${ }_{5}$ School of Textiles, Ieungham Lniversity, Gyeongsan 712-749, Korea \\ Received December 27, 2007
}

Key Words : Pỵranobenzophenone. Clusiacitran A. Vismiaphenone B. Isovismiaphenone B. Mỵrtiaphenone B

Molecules with pyranobenzophenone moiety are widely distributed in nature. ' They have a range of biological and pharmacological properties. ${ }^{2}$ Among these, clusiacitran A (1) with a citran nucleus was isolated as an optically inactive racentate along with clusiacitran $B(2)$ from an extract of the fruits of Chisia miltiflora (Figure 1). ${ }^{3}$ Recently. clusiacitran A (1) was also isolated from Gatcinia schombrigkiana Pierre, which was collected from Songkla area in southern Thailand along with clusiacitran $B$ (2) and fluorinated clusiacitrans A (3) and B (4). "This plant is used in the treatment of coughs and diabetes as well as for improving the menstrual blood quality. The crude hexane extract of the stems of this plant has also shown antimalarial activity with an $\mathrm{EC}_{50}$ value of $2.2 \mu \mathrm{g} \mathrm{mL} \mathrm{m}^{-1}$. Although the structires of these materials $1-t$ have been determined by spectral and $X$ ray analysis, ${ }^{+}$there are no synthetic approaches to these conpounds

Both vismiaphenone B (5) and isovismiaphenone B (6) with prenylated benzophenone moiety were isolated from the fruits of either Vismia decipiens or Chisia ellipticifolia ${ }^{\circ}$ Although one synthetic approach to vismiaphenone B (5) and isovismiaphenone $B(6)$ has been reported. this synthetic route is linited by its many reaction steps, harsh reaction conditions, and low yield involving many side reactions. ${ }^{7}$ Myrtiaphenone B (7) was isolated from both Garcinia mwifolia and Garinia psendoguttifera ${ }^{8}$ The biosynthetic approaches to myrtiaphenone B (7) have been described, but there are no reports on the synthesis of compound $7 .^{8.9}$

Recently. we reported a new methodology for preparing citrans and cyclols with polycycles by ethylenediamine diacetate (EDDA)-catalyzed reactions of substituted trihydroxybenzenes to citral or transtrans-farnesal. ${ }^{10}$ We also reported a new methodology for synthesizing benzopyrans wa ethylenediamine diacetate (EDDA)-catalyzed reactions of resorcinols to $\alpha \beta$-unsaturated aldehydes. ${ }^{13}$ As a part of an ongoing study into the synthetic efficacy of these two methodologies, this study examined the synthesis of naturally occurring molecules with the pyranobenzophenone moiety. This paper reports an efficient and concise synthesis of clusiacitran A (1). vismiaphenone B (5), isovismiaphenone B (6). and nyrtiaphenone B (7).

\section{Results and Discussion}

Scheme I shows the retrosynthetic strategy. The synthesis of natural clusiacitran A (1) could be prepared using tandem electrocyclization of 2.4,6-trilydrosybenzophenone (9) and citral using the method previously developed by our group. ${ }^{10}$ Compound 9 could be prepared from conmercially available phloroglucinol (8) using a Friedel-Crafts acylation reaction. l? $^{2}$

Reaction of plloroglucinol (8) with benzoyl chloride in the presence of $\mathrm{AlCl}_{3}$ gave compound 9 in $48 \%$ yield (Scheme 2). ${ }^{12}$ The treatment of compound 9 with 2.0 equiv of citral in the presence of $20 \mathrm{~mol} \%$ of EDDA at $100^{\circ} \mathrm{C}$ for $10 \mathrm{~h}$ in DMF afforded naturally occurring compound 1 in $54 \%$ yield as a sole product without any expected regio-

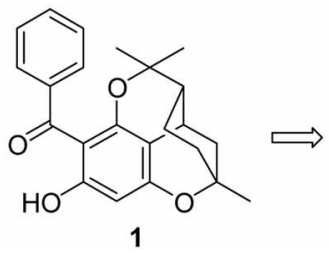<smiles>O=C(c1ccccc1)c1c(O)cc(O)cc1O</smiles>

Scheme 1

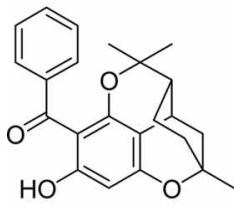

1

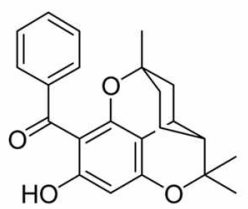

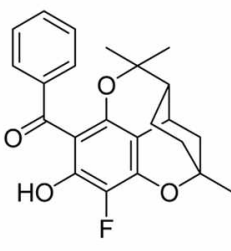

3

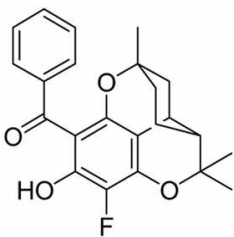

4

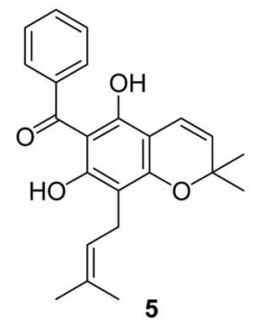

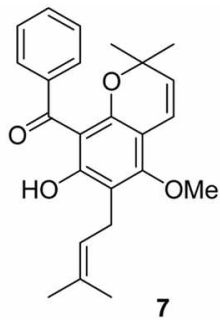

Figure 1. Selected naturally occuring molecules with benzophenone moiety. 


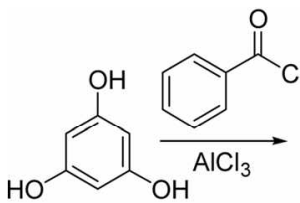

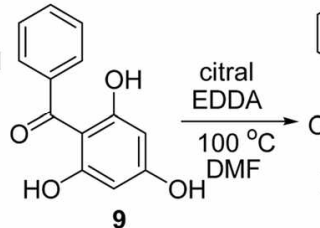

Scheme 2

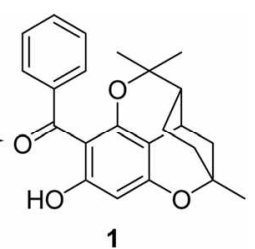

1

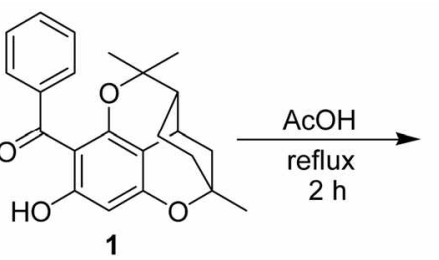<smiles>C=C(C)C1CCC2(C)CC1c1c(cc(O)c(C(=O)c3ccccc3)c1O)O2</smiles>

Scheme 3

The synthesis of vismiaphenone B (5) and isovisnmiaphenone B (6) was next attempted as shown in Scheme 4. The C-prenylation of 2,4,6-trilydroxyacetophenone with prenyl bromide in $\mathrm{KOH}$ solution has been reported. However, following this method. the prenylated product 17 was only produced in $30 \%$ yield. In order to increase the yield. the base was changed to DBU. Interestingly, a reaction of 2,4.6trihydrobenzophenone (9) with 1-bromo-3-methyl-2-butene in the presence of DBU in THF at room temperature for $24 \mathrm{~h}$ afforded the prenylated benzopheneone 17 in $42 \%$ yield. Treatment of compound 17 with 3-methyl-2-butenal in the presence of $20 \mathrm{~mol} \%$ of EDDA in methylene chloride at room temperature for $5 \mathrm{~h}$ provided the naturally occurring products 5 and 6 in 31 and $51 \%$ yields, respectively. The assignment of compounds 5 and $\mathbf{6}$ was made by a comparison with the data reported for the natural products. ${ }^{5.7}$ The ${ }^{1} \mathrm{H}$ NMR spectrum of compound 5 showed signals of two vinylic protons on $2 H$-pyranyl ring at $\delta=6.58$ (d. $J=10.0$ $\mathrm{Hz})$ and $5.47(\mathrm{~d}, J=10.0 \mathrm{~Hz})$. whereas that of compound 6 showed signals of two viny lic protons at $\delta=6.45(\mathrm{~d}, J=10.0$ $\mathrm{Hz})$ and $5.26(\mathrm{~d}, J=10.0 \mathrm{~Hz})$.

Finally, the synthesis of natural product myrtiaphenone $B$ (7) was attempted by a metlyylation reaction of compound 6 . A reaction of compound 6 with dimethylsulfate in the presence of $\mathrm{K}_{2} \mathrm{CO}_{3}$ in acetone at room temperature for $1 \mathrm{~h}$ produced the natural product 7 and its derivative 18 in 75 and $11 \%$ yield. respectively. The spectroscopic data of the synthetic material 7 was same as that reported in the literature.

In conclusion, a new synthetic route for biologically interesting natural products clusiacitran $A(1)$. visniaphenone $B$ (5). isovismiaphenone B (6), and myrtiaphenone B (7) with a pyranobenzophenone moiety was developed starting from phloroglucinol. The key strategy in this synthetic route is a Freidel-Crafts acylation followed by EDDA-catalyzed electrocyclization. The acid-catalyzed cleavage reaction of compound 1 was described as a model study for the syinthesis of other natural products with oxabicyclononane moiety:

Figure 2. Selected naturally occumng molecules with oxabicyclononane moiety.<smiles>CC(=O)/C(C)=C\CBr</smiles>

9<smiles>CC(C)=CCc1c(O)cc(O)c(C(=O)c2ccccc2)c1O</smiles><smiles>CC(C)=CC=O</smiles><smiles>CC(C)=CCc1c(O)c(C(=O)c2ccccc2)c(O)c2c1OC(C)(C)C=C2</smiles><smiles>CC(C)=CCc1c(O)c(C(=O)c2ccccc2)c(OC(C)(C)C)c2c1C=CC(C)(C)O2</smiles>

Scheme 4 


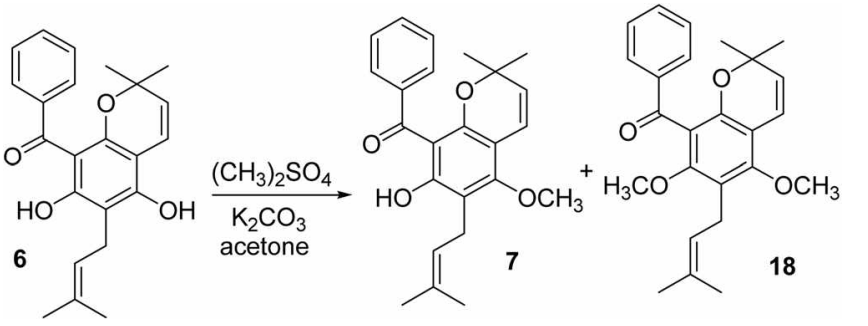

Scheme 5

The synthesis of natural products with an oxabicyclononane skeleton is currently underway in our laboratory

\section{Experimental Section}

All the experiments were carried out in a nitrogen atmosphere. Merck precoated silica gel plates (Art. 5554) with a fluorescent indicator were used for analytical TLC. Flash column chromatography was performed using silica gel 9385 (Merck). The ${ }^{1} \mathrm{H}$ NMR and ${ }^{13} \mathrm{C}$ NMR spectra were recorded on a Bruker Model ARX (300 and $75 \mathrm{MHz}$. respectively) spectrometer in $\mathrm{CDCl}_{3}$ or $\mathrm{DMSO}_{-\mathrm{d}}$. The IR spectra were recorded on a Jasco FTIR 5300 spectrophotometer. The MS spectra were carried out at the Korea Basic Science Institute.

2,4,6-Trihydroxybenzophenone (9). Alumium trichloride $(5.334 \mathrm{~g} .40 .0 \mathrm{mmol})$ was added to a stirred mixture of phloroglucinol (8) (1.261 g. $10.0 \mathrm{mmol}$ ) in nitrobenzene (50 $\mathrm{mL}$ ) and stirred for $30 \mathrm{~min}$ at roon temperature. Then. benzoyl chloride ( $1.406 \mathrm{~g}, 10.0 \mathrm{mmol}$ ) was injected and heated to $60^{\circ} \mathrm{C}$ for $3 \mathrm{~h}$, and the reaction mixture was cooled to room temperature. The mixture was poured onto ice water and extracted with ethyl acetate $(3 \times 50 \mathrm{~mL})$. The organic layer was washed with $1 \mathrm{~N} \mathrm{NaOH}$ solution. water $(2 \times 30$ $\mathrm{mL})$. and brine $(2 \times 30 \mathrm{~mL})$. The organic layer was dried $\left(\mathrm{MgSO}_{4}\right)$. filtered. and evaporated to give residue. The residue was purified by column chromatography on silica gel using hexane/ethylacetate $(5: 1)$ to give $9(1.105 \mathrm{~g} .48 \%$ ) as a solid: mp $169-170^{\circ} \mathrm{C}:{ }^{1} \mathrm{H}$ NMR $\left(300 \mathrm{MHz}, \mathrm{DMDO}-\mathrm{d}_{6}\right)$ $\delta 10.08(1 \mathrm{H}, \mathrm{s}) .9 .82(\mathrm{lH} . \mathrm{s}) .7 .61(2 \mathrm{H} . \mathrm{m}) .7 .5 \mathrm{l}(\mathrm{lH}, \mathrm{t}, J=$ $7.4 \mathrm{~Hz}) .7 .43(1 \mathrm{H}, \mathrm{t} . J=7.7 \mathrm{~Hz}) .5 .84(2 \mathrm{H} . \mathrm{s}):{ }^{13} \mathrm{C}$ NMR $(75$ MHz. DMDO-d 6 ) $\delta 196.5,161.9 .159 .4,139.9 .131 .8,128.5$. 128.0. 105.7, 94.4: IR (KBr) 3371. 3267, I642. 1579, 1572. 1470, 1451, 1323, 1290, 1240, 1154, 1060,920,824, 756 . $700 \mathrm{~cm}^{-1}$ : EIMS nu/z (\%) $230\left(\mathrm{M}^{-}, 50\right), 229(100), 153(38)$. $152(5), 105(9) .77(13)$

Clusiacitran A (1). To a solution of $9(0.23 \mathrm{~g} .1 .0 \mathrm{mmol})$ and citral $(0.304$ g. $2.0 \mathrm{mmol})$ in DMF $(20 \mathrm{~mL})$ was added ethylenediamine diacetate $(0.036 \mathrm{~g} .0 .2 \mathrm{mmol})$ at room temperature. The reaction mixture was heated to $100^{\circ} \mathrm{C}$ for $10 \mathrm{~h}$ and then cooled to room temperature. Evaporation of solvent and purification by columu chromatography on silica gel using hexane/ethỵlacetate $(15: 1)$ gave $1(0.197 \mathrm{~g}$. $54 \%$ ) as a solid: mp 211-213 ${ }^{\circ} \mathrm{C}$; ${ }^{~} \mathrm{H}$ NMR $(300 \mathrm{MHz}$. $\left.\mathrm{CDCl}_{3}\right) \delta 12.64(\mathrm{lH} . \mathrm{s}), 7.60-7.32(5 \mathrm{H}, \mathrm{m}), 6.10(\mathrm{lH}, \mathrm{s})$. $2.70-2.53(\mathrm{lH}, \mathrm{ml}) .2 .22-2.12(2 \mathrm{H}, \mathrm{nl}) .1 .92-1.85(2 \mathrm{H}, \mathrm{ml})$. $1.48-1.38(1 \mathrm{H}, \mathrm{m}), 1.37(3 \mathrm{H}, \mathrm{s}), 1.27-1.18(1 \mathrm{H}, \mathrm{m}), 1.08$
(3H. s), 0.8-0.7 (lH, m), 0.59 (3H. s): ${ }^{13} \mathrm{C} \mathrm{NMR} \mathrm{(75} \mathrm{MHz,}$ $\left.\mathrm{CDCl}_{3}\right) \delta 198.1,164.3,163.2,159.2 .142 .1 .130 .3 .127 .6$. $127.1,197.8,107.3,97.2 .85 .7,76.1,45.6,37.4 .34 .6,29.1$, 28.5. 27.6. 23.4. 21.5; IR (KBr) 3455. 2975, 2926. 1624, 1562. 1478. 1352. 1323. 1308. 1165. 1140. 1073. 1042.951, $880,820.704 \mathrm{~cm}^{-1}$. EIMS n/z (\%) $364\left(\mathrm{M}^{+}, 45\right), 349(13)$, 321 (5), 283 (14), 282 (31). 281 (100), 203 (16), 105 (33). 77 (29).

Compound 16. Compound 1 (0.073 g. $0.2 \mathrm{mmol})$ in acetic acid $(5 \mathrm{~mL})$ was refluxed for $2 \mathrm{~h}$ and then cooled to room temperature. Water $(30 \mathrm{~mL})$ was added and the solution was extracted with ethyl acetate $(3 \times 30 \mathrm{~mL})$. The organic layer was washed saturated sodium bicarbonate solution $(2 \times 30 \mathrm{~mL})$ and brine $(30 \mathrm{~mL})$. The organic layer was dried $\left(\mathrm{MgSO}_{4}\right)$, filtered, and evaporated to give residue. The residue was purified by columu chromatography on silica gel using hexane/etlyylacetate $(10: 1)$ to give $16(0.062$ g. $85 \%$ ) as a solid: $\operatorname{mp~} 149.150{ }^{\circ} \mathrm{C}:{ }^{1} \mathrm{H}$ NMR $(300 \mathrm{MHz}$, $\left.\mathrm{CDCl}_{3}\right) \delta 8.86(\mathrm{lH} . \mathrm{s}), 7.6-7.43(5 \mathrm{H} . \mathrm{m}), 6.11$ (lH. s), 4.63 (lH. s). 4.42 (lH. s). $3.50-3.40$ (lH, m). 2.44-2.25 (lH. m), $2.00-1.45(6 \mathrm{H}, \mathrm{m}), 1.8 \mathrm{l}(3 \mathrm{H}, \mathrm{s}), 1.37(3 \mathrm{H} . \mathrm{s}) ;{ }^{12} \mathrm{C}$ NMR $(75$ $\left.\mathrm{MHz}, \mathrm{CDCl}_{3}\right) \delta 197.2,164.3 .160 .0,159.8 .148 .2,140.1$ $131.8,128.9,128.1$. 109.5, 103.7. 95.6. 76.4. 48.2. 39.2. 37.3, 29.8, 28.4. 23.0. 22.8: IR (KBr) 2932. 1626, 1449. 1304. 1167, 1136. 1090. 883. $822.739 \mathrm{~cm}^{-1}$ : EIMS m/z (\%) $364\left(\mathrm{M}^{-}, 19\right) .349(5), 321(5), 281$ (100). 203 (12). $105(17)$. 77 (19)

3-Prenyl-2,4,6-trihydroxybenzophenone (17). A mixture of $9(0.276 \mathrm{~g}, 1.2 \mathrm{mmol})$, prenyl bromide $(0.179 \mathrm{~g} .1 .2$ mmol). and DBU (0. $183 \mathrm{~g}, 1.2 \mathrm{mmol})$ in dry THF (10 mL) was stirred at room temperature for $24 \mathrm{~h}$. Addition of $2 \mathrm{~N}$ $\mathrm{HCl}$ solution $(30 \mathrm{~mL})$. and extraction with ethyl acetate $(3 \times$ $30 \mathrm{~mL}$ ). washing with brine $(30 \mathrm{~mL})$, drying over $\mathrm{MgSO}_{4}$ and removal of the solvent followed by flash column chromatography on silica gel using hexane/ethylacetate (7:1) gave $17\left(0.151\right.$ g. $42 \%$ ) as an oil: ${ }^{1} \mathrm{H}$ NMR (300 MHz, $\left.\mathrm{CDCl}_{3}\right) \delta 10.3(\mathrm{lH}, \mathrm{s}), 7.63-7.45(5 \mathrm{H}, \mathrm{m}) .6 .42(\mathrm{lH}$, br s). 5.90 (1H. s). $5.23-5.20(1 \mathrm{H}, \mathrm{m}) .3 .32(2 \mathrm{H} . \mathrm{d} . J=7.1 \mathrm{~Hz}$ ). $1.77(3 \mathrm{H}, \mathrm{s}) .1 .73(3 \mathrm{H}, \mathrm{s}),{ }^{13} \mathrm{C}$ NMR $\left(75 \mathrm{MHz}, \mathrm{CDCl}_{3}\right) \delta$ 197.5, 162.0, 161.5. 158.3. 140.1, 132.0, 129.0. 127.7 . $103.8,101.7 .96 .8 .32 .0 .26 .7,16.1:$ IR (neat) 3389, 2977. 2924. 1625, 1516, 1449, 1321, 1383, 1321, 1176, 1119. 1074. 821. $736 \mathrm{~cm}^{-1}$; EIMS m/z (\%) $298\left(\mathrm{M}^{-}, 100\right), 297$ (24). $283(32) .255(20), 243(90), 241(36), 229(20) .205$ (18). $165(71) .129(21) .77(23)$.

Vismiaphenone B (5) and isovismiaphenone B (6). To a solution of $17(0.089 \mathrm{~g} .0 .3 \mathrm{mmol})$ and 3-methyl-2-butenal $(0.051 \mathrm{~g} .0 .6 \mathrm{mmol})$ in methylene chloride $(10 \mathrm{~mL})$ was added ethylenediamine diacetate $(0.011 \mathrm{~g} .0 .06 \mathrm{mmol})$ at room temperature. The reaction mixture was stirred at room temperature for $5 \mathrm{~h}$. Water $(30 \mathrm{~mL})$ was added and the solution was extracted with methy lene chloride $(3 \times 30 \mathrm{~mL})$. Evaporation of solvent and purification by column chromatography on silica gel using hexane/ethylacetate $(20: 1)$ gave products $5(0.034 \mathrm{~g} .31 \%)$ and $6(0.056 .51 \%)$. Compound 5 : ${ }^{1} \mathrm{H}$ NMR $\left(300 \mathrm{MHz}, \mathrm{CDCl}_{3}\right) \delta 9.19(\mathrm{lH}, \mathrm{s}) .8 .72(\mathrm{lH}, \mathrm{s})$, $7.63-7.47(5 \mathrm{H} . \mathrm{m}) .6 .58(1 \mathrm{H}, \mathrm{d} . J=10.0 \mathrm{~Hz}), 5.47(\mathrm{lH} . \mathrm{d} . J$ 
$=10.0 \mathrm{~Hz}) \cdot 5 \cdot 15(1 \mathrm{H}, \mathrm{t}, J=7.0 \mathrm{~Hz}) \cdot 3.24(2 \mathrm{H}, \mathrm{d} . J=7.0 \mathrm{~Hz})$. $1.74(3 \mathrm{H}, \mathrm{s}), 1.66(3 \mathrm{H}, \mathrm{s}), 1.43(6 \mathrm{H}, \mathrm{s}):{ }^{13} \mathrm{C} \mathrm{NMR}(75 \mathrm{MHz}$. $\left.\mathrm{CDCl}_{3}\right) \delta 197.5$. 159.3. 159.0, 155.1, 140.0, 132.5. 132.2. 129.2. 127.8. 127.1, 125.8. 122.1, 116.1, 108.4, 104.5. 102.4. 77.9. 28.4, 25.8, 21.4. 17.9: IR (neat) 3503, 2976. 2924. 1616, 1449, 1377. 1323. 1177. 1134. $885.746 \mathrm{~cm}^{-1}$ : EIMS m/z (\%) $364\left(\mathrm{M}^{+} .43\right) .350(24), 349(100), 347(13)$. 309 (12). 294 (16). 293 (80). 215 (30). 105 (28). 77 (14).

Compound 6: mp 119-120 ${ }^{\circ} \mathrm{C}$ : ${ }^{1} \mathrm{H}$ NMR $(300 \mathrm{MHz}$. $\left.\mathrm{CDCl}_{3}\right) \delta \mathrm{I} 2.80(\mathrm{lH}, \mathrm{s}) \cdot 7.46-7.35(5 \mathrm{H}, \mathrm{m}) .6 .45(\mathrm{lH} . \mathrm{d}, J=$ $10.0 \mathrm{~Hz}), 6.34(\mathrm{lH}, \mathrm{s}) .5 .30(\mathrm{lH} . \mathrm{t} . J=7.0 \mathrm{~Hz}) .5 .26(\mathrm{lH} . \mathrm{d} . J$ $=10.0 \mathrm{~Hz}), 3.41(2 \mathrm{H}, \mathrm{d}, J=7.0 \mathrm{~Hz}), 1.84(3 \mathrm{H}, \mathrm{s}) .1 .78(3 \mathrm{H}$. s). $0.95\left(6 \mathrm{H}\right.$. s): ${ }^{13} \mathrm{C}$ NMR $\left(75 \mathrm{MHz} . \mathrm{CDCl}_{3}\right) \delta 200.5 .162 .0$. 157.9. 154.5. 142.9, 136.7, 129.9. 127.5, 127.1, 125.2 . 121.7. 115.9, 105.3. 105.0, 101.8, 77.3, 27.3, 25.9. 21.7. 17.9: IR (KBr) 3432, 2924, 1601. 1422, 1319, 1275, 1169. $1136.817 \mathrm{~cm}^{-1}$ : EIMS $\mathrm{m} / \mathrm{z}(\%) 364\left(\mathrm{M}^{+}, 52\right) .350(24) .349$ (100). $347(11), 294(20), 293(99), 215(35), 129(19), 105$ (19), $77(11)$.

Myrtiaphenone B (7) and its derivative 18. $\mathrm{K}_{2} \mathrm{CO}_{3}$ $(0.097 \mathrm{~g} .0 .70 \mathrm{mmol})$ was added to a solution of $6(0.05 \mathrm{lg}$. $0.14 \mathrm{mumol})$ in acetone $(10 \mathrm{~mL})$ at room temperature. Dimethyl sulfate $(0.018 \mathrm{~g}, 0.14 \mathrm{~mm}$ ol) was then added dropwise, and the mixture was stirred at room temperature for $\mathrm{l} \mathrm{h}$. A saturated $\mathrm{NH}_{4} \mathrm{Cl}$ solution $(30 \mathrm{~mL})$ was added and the mixture was extracted with ethyl acetate $(3 \times 30 \mathrm{~mL})$. The organic layer was dried over $\mathrm{MgSO}_{4}$ and the solvent was removed under reduced pressure to leave an oily residue. The residue was then purified by columun chromatography on silica gel using hexane/ethyl acetate $(30: 1)$ to give products $7(0.040$ g. $75 \%)$ and $18(0.006$ g. $11 \%)$. Compound 7: ${ }^{1} \mathrm{H} \mathrm{NMR}\left(300 \mathrm{MHz}, \mathrm{CDCl}_{3}\right) \delta 12.03(\mathrm{lH}, \mathrm{s}), 7.50-$ $7.33(5 \mathrm{H}, \mathrm{m}), 6.40(1 \mathrm{H}, \mathrm{d} . J=10.0 \mathrm{~Hz}), 5.31(1 \mathrm{H}, \mathrm{d}, J=10.0$ $\mathrm{Hz}), 5.24(1 \mathrm{H}, \mathrm{t}, J=6.9 \mathrm{~Hz}) .3 .76(3 \mathrm{H}, \mathrm{s}), 3.30(2 \mathrm{H} . \mathrm{d}, J=$ $6.9 \mathrm{~Hz}) .1 .78(3 \mathrm{H} . \mathrm{s}), 1.69(3 \mathrm{H} . \mathrm{s}) .0 .97(6 \mathrm{H}, \mathrm{s}):{ }^{13} \mathrm{C}$ NMR $\left(75 \mathrm{MHz}, \mathrm{CDCl}_{3}\right) \delta 200.6,162.0,160.6,158.0 .142 .4,131.5$. 130.4. 127.5, 127.3. 126.0. 123.8, 116.8, 114.7, 111.5, 107.9. 77.0.62.0. 27.3. 25.8. 22.2. 17.9: IR (neat) 2926. 1599. 1419. 1389. 1296. 1215. 1171. 1136. 1111.966, $752 \mathrm{~cm}^{-1}$ : EIMS m/z (\%) $378\left(\mathrm{M}^{+} .30\right) .363(100) .307(20), 323(86)$. $105(70)$.

Compound 18: ${ }^{\mathrm{H}} \mathrm{H}$ NMR $\left(300 \mathrm{MHz}, \mathrm{CDCl}_{3}\right) \delta 7.87-7.84$ $(2 \mathrm{H}, \mathrm{m}), 7.55-7.50(1 \mathrm{H}, \mathrm{m}), 7.43-7.38(2 \mathrm{H} . \mathrm{m}), 6.50(\mathrm{lH}, \mathrm{d}$. $J=10.0 \mathrm{~Hz}) .5 .50(1 \mathrm{H}$. d. $J=10.0 \mathrm{~Hz}) .5 .18(1 \mathrm{H} . \mathrm{t} . J=6.7$ Hz) $3.75(3 \mathrm{H} . \mathrm{s}) .3 .60(3 \mathrm{H} . \mathrm{s}) .3 .27(2 \mathrm{H} . \mathrm{d} . J=6.7 \mathrm{~Hz}) .1 .73$ (3H. s). 1.67 (3H. s) 1.17 (6H. s): IR (neat) $2975,1674$. 1580, 1451, 1418, 1316, 1283, 1215, 1128, 1100, 1005, 966. $889.729 \mathrm{~cm}^{-1}$ : EIMS m/z (\%) $392\left(\mathrm{M}^{-} .10\right), 368(34), 286$ (45), 284 (61), 277 (89). 197 (66). 106 (58), 78 (100). 69 (48). $57(51) .55(42)$

Acknowledgment. This work was supported by grant No.
RTI04-01-04 from the Regional Technology Innovation Program of the Ministry of Conmerce Industry and Energy (MOCIE).

\section{References}

1. (a) Bernardi, A. P. M.: Ferraz, A. B. F; Albring. D. V: Bordignon. A. A. L.: Schripsema. T.: Bridi. R.: Dutra-Filho. C. S.: Henriques. A. T.: von Poser. G. L. J. Nat. Prod. 2005. 68. 784. (b) McKee. T. C.: Covington. C. D.: Fuller. R. W.: Bokesch. H. R.: Young. S.: Cardellina, J. H.. II; Kadushin, M. R.; Soejarto. D. D.: Stevens. P. F.: Cragg. G. M.; Boyd, M. R. J. Kat Prod. 1998. 61, 1252. (c) Cao. S.-G.: Wu, X.-H.: Sim, K.-Y; Tan, B. H. K.: Vittal, J. J:; Pereira. J. T.: Goh. S.-H. Heh: Chim. Acta 1998. 81. 14(44. (d) Cao. S.-G.: Siml. K.-Y.: Pereira. T.: Goh. S.-H. Phwochemistry 1998. 47. 1051. (e) Cao. S. G.: Chong. K. L.: Vittal. J. J: Sim. K. Y.: Goh. S. H. Nat. Prod. Lett. 1998. 11. 233. (f) Gustafson. K. R.: Bokesch. H. R.: Fuller. R. W.: Cardellina, J. H.. II: Kadushin. M. R.: Soejarto. D. D.: Boyd, M. R. Tetrahedron Lett. 1994. 35. 5821 .

2. (a) Ito. C.: Itoigawa. M.: Mishina. Y.: Filho. V. C.: Enjo. F.: Tokuda. H.: Nishino. H.: Furukawa. H. J. Nat. Prod. 2003. 66 . 368. (b) Fuller. R. W.: Blunt, J. W: Boswell, J. L.: Cardellina. J. H., II: Bovd, M. R. J. Kat Prod. 1999. 62, 130. (c) Fuller. R. W: Westergaard, C. K.; Collins. J. W.; Cardellina. J. H.s II; Boyd, M. R. J. Nat. Prod 1999. 62.67. (d) Chaturvedula. V. S. P.: Schilling. J. K.: Kingston. D. G. I. J. Kat. Prod. 2002. 65. 965. (e) Prado. S.: janin. Y. L.: Saint-Joanis. B.: Brodin. P.: Michel. S.: Koch. M.: Cole. S. T.: Tillequin. F.: Bost. P.-E. Bioorg. Med Chem. 2007. 15. 2177.

3. Gonzalez, J. G.: Olivares. E. M:; Monache, F. D. Phvtochemisty $1995.38,485$

4. Fun. H.-K: Ky somboon. S.: Chantrapromma. K.: Chantrapromma. S. Acta Crrst. 2006. E62.03228.

5. Monache. G. D.: Gonzalez. T. G.: Monache. F. D.: Bettolo. G. B M. Phutochemisov 1980, 19. 2025.

6. Gonzalez, J. G: Cuellar. V.: Betancourt. A.: Pinzon. M. I. Phytochemistry 1983. 22, 2088

7. Pathak. V. P.: Khanna. R. N. Bull. Chem. Soc. Jpm. 1982. 55. 2264.

8. Spino. C.: Lal. J.: Sotheeswaran. S: Aalbersherg. W. Ptntachemism 1995.38.233

9. Ali. S.: Goundar, R; Sotheeswaran, S: Beaulier. C.: Spino. C. Phutochemisoy, 2000, $53,281$.

10. Lee. Y. R.; Kim. J. H. Synlett 2007. 2232

11. (a) Lee. Y. R.: Choi. J. H.: Yoon. S. H Tetrahedron Lett. $2005 .+6$. 7539. (b) Lee. Y. R.: Wang. X. Bull. Korean Chent. Soc. 2005. 26. 1933. (c) Lee. Y. R.: Xia. L. Bull. Korew Chen. Soc. 2007. 28. 1585. (d) Wang. X.: Lee, Y. R. Synthesis 2007, 3044. (e) Lee, Y. R; Xia. X. Symhesis 2007, 3244. (f) Wang. X; Lee. Y. R. Tetrahedron Lett. 2007, 48. 6275. (g) Lee. Y. R.: Li. X. Bull. Korew Chem. Soc. 2007. 28. 1739.

12. Lin. C.-M.: Huang. S.-T:: Lee. F.-W: Kuo. H.-S.: Lin. M.-H. Bioorg. Med Chem. 2006. 1t. 4402

13. Calderon-Higginson. C.: Crombie, L.: Redshaw: S. D.: Whiting D. A. J Chem Soc. Perkin Trans. I 2000. 2491

14. Shoyama. Y: Morimoto. S.; Nishioka. I. Chem. Pham. Bull. 1981. 29.3720.

15. (a) Wu. T.-S.: Wang. M.-L.: Wu. P.-L.: Jong. T.-T. Phtochemistry 1995. 40. 1817. (b) Wu. T.-S.: Wang. M.-L.: Wu. P.L. Plntochemisty $1996 .+3,785$. 